\title{
Reaction Zones and Quenched Charged-Particle Systems with Long-Range Interactions
}

\author{
A.D. Rutenberg \\ Centre for the Physics of Materials, Physics Department, McGill University, \\ 3600 rue University, Montréal QC, Canada H3A $2 T 8$
}

(October 30, 2018)

\begin{abstract}
We determine the evolving segregated or mixed morphology of charged-particle systems with longrange power-law interactions and overall charge neutrality that have been quenched to a low temperature. Segregated morphology systems are characterized by the size of uniformly charged domains, $L(t)$, the particle separation within the domains, $l_{A A}(t)$, the particle flux-density leaving the domains, $J(t)$, the width of reaction zones between domains, $W(t)$, the particle spacing within the reaction-zones, $l_{A B}(t)$, and the particle lifetime in the reaction-zones, $\tau(t)$. Mixed morphology systems are essentially one large reaction zone, with $L \sim l_{A B} \sim l_{A A}$. By relating these quantities through the scaling behavior of particle fluxes and microscopic annihilation rates within reactionzones, we determine the characteristic time-exponents of these quantities at late times. The morphology of the system, segregated or mixed, is also determined self-consistently. With this unified approach, we consider systems with diffusion and/or long-range interactions, and with either uncorrelated or correlated high-temperature initial conditions. Finally, we discuss systems with particle-like topological defects and electronic systems in various substrate dimensions - including quantum-hall devices with skyrmions.
\end{abstract}

\section{INTRODUCTION}

Reaction-diffusion systems without long-range interactions [1 8] involve two diffusing species of particles that annihilate on contact, $A+B \rightarrow \emptyset$. The temperature is assumed to be low enough that the reverse reaction can be ignored, so that the densities at late times are determined by the uncorrelated initial conditions. For equal initial mean-densities of the particles and anti-particles, there are two possible morphologies at late times [1]. The first, for spatial dimension $d \geq 4$, is a well mixed morphology in which mean-field dynamics applies to the particle density, $\partial_{t} \bar{\rho}=-C \bar{\rho}^{2}$. This leads to $\bar{\rho}(t) \sim 1 / t$ at late times. The second, for $d<4$, is a segregated morphology consisting of single-species domains of characteristic size $L(t)$. The evolution is via diffusive currents feeding particles from the domains into reaction zones where they are annihilated by anti-particles. The evolution of the mean domain density, $\bar{\rho} \sim t^{-d / 4}$, is easily demonstrated when the two species have equal diffusion constants, so that the density difference $\Delta \rho \equiv \rho_{A}-\rho_{B}$ satisfies a linear diffusion equation [1,2]. It also applies more generally [3].

In the mixed morphology initial fluctuations decay faster than the mean-density and can be ignored. In the segregated morphology, long-wavelength initial fluctuations decay more slowly and asymptotically determine the domain structure. In that case, the profile of the domains and the structure of the reaction-zone can be understood through the particle fluxes and the annihilation kinetics of particle pairs 田 [7, as well as on a more formal level [3].

It has been recognized from the beginning [1] that long-range interactions between oppositely-charged particles and antiparticles change the evolution of a reaction- diffusion system - both by changing the initial chargedensity fluctuations, and by changing the subsequent dynamics. However, progress has been gradual due to the greater complexity of long-range interactions both in analytical models [9] and in computer simulations, and by the need to understand the force-free case first. Nevertheless, progress in long-range models has been made along a number of fronts 10 18]. For systems with long-range interactions but no diffusion, and with uncorrelated initial conditions, Toyoki presented an analysis of a mixed morphology [10]. Complementing that work, Ispolatov and Krapivsky [11 considered segregated systems and simulated a variety of force-laws in $d=1$. For both longrange interactions and diffusion, with uncorrelated initial conditions, a self-consistent model by Ginzburg et al. 12] and a complementary scaling model 13 has captured the density evolution for $n \geq d-1$, where $n$ characterizes the force between particles via $f \sim r^{-n}$ (see Eqn. (11) below). The $n=d-1$ Coulombic case has also been treated by Ohtsuki [14]. High-temperature correlated initial conditions have also been discussed 15.

In this paper, we are interested in the late-time evolution of initially random distributions of charges in homogeneous systems with overall charge neutrality. We use the scaling behavior, with respect to length-scale, of the initial charge fluctuations, the resulting large-scale currents, and the local annihilation rate of particles to determine the morphology as well as the time-exponents of the characteristic lengths. Our approach is based on the assumption that domain structures are characterized by only two lengths: their size $L(t)$ and their characteristic particle separation, $l_{A A}(t)$, where the average density $\bar{\rho} \sim l_{A A}^{-d}$. This is comparable to the dynamical-scaling assumption of phase-ordering systems 19] and, with appro- 
priate physical input, leads to a self-consistent description of the system evolution. Between the domains, we allow for reaction zones of width $W(t)$, within which both particle species are mixed with typical spacing $l_{A B}(t)$ and lifetime $\tau$. For segregated systems, charged currents are absorbed in the reaction zones. For mixed systems, the reaction-zone pervades the system. Our approach of balancing currents with annihilation within reaction zones is in the spirit of the treatment of force-free systems by Redner and Leyvraz [7]; and for these systems our results agree with the Renormalization-Group analysis of Lee and Cardy [3].

We ignore correlations apart from the characteristic lengths $l_{A A}, l_{A B}, L$, and $W$ which determine the densities and sizes of the domains and reaction zones. As a result, our approach is insensitive to early-time dynamics, unequal diffusion constants [20], and motion of domain boundaries. All of these factors help determine the amplitude of the growth laws. Nonetheless, from the scaling properties of currents and lengths we can extract the asymptotic time-exponents. One benefit of our approach is that its conclusions are robust to the details of the system, and that it provides a vividly physical picture of the morphological evolution of charged systems with long-range interactions.

We should emphasize what is new in this paper. We do not assume which morphology the system selects, but determine it self-consistently from the physics. We treat a large number of different cases with the same unified approach, including the well understood diffusion-only systems. We determine the reaction zone width and density, and also discover non-trivial domain-edge profiles. For uncorrelated initial conditions, we discover several regimes where ballistic annihilation is the dominant annihilation mechanism. For high-temperature equilibrium initial conditions, we determine the appropriate initial charge-fluctuation spectrum and show how it modifies the subsequent evolution and morphology of the system.

In the next section we introduce long-range interactions with overdamped dynamics between initiallyrandom particles and anti-particles. For equilibrium initial conditions, we calculate the expected initial long-wavelength charge fluctuations, characterized by $\mu$ [Sec. II A, see also Fig. 1]. From the charge fluctuations, using a scaling form for the domain profiles near edges, we determine the scaling of charged currents [Sec. IIB, see also Tab. If and Fig. 2]. We then consider various mechanisms of particle annihilation [Sec. IIC, see also Figs. 3 and 4 . We combine our results on currents and on annihilation, by balancing the currents with the annihilation rates in the reaction zones [Sec. IID. This is sufficient to determine both the system morphology, and the time dependence of the domain size $L$ and of the average density, $\bar{\rho}$ [Fig. 5 and Tab. II]. We next consider the reaction zone in more detail, and determine its width $W$ and particle spacing $l_{A B}$, as well as the typical lifetime $\tau$ within the reaction zone [Sec. IIE, see also Tab. III]. Finally we discuss our results, including implications for coarse-grained treatments [Sec. II F].

We discuss the previous reaction-diffusion literature [Sec. III], then discuss applying our results to electrically charged $(n=2)$ systems in various substrate dimensions [Sec. IV]. We emphasize that for $d<3$ novel decay laws and segregated morphologies are found. We then discuss the application of our results to quenched phase-ordering systems with point-like topological defects [Sec. V]. We can also extend our approach to include Lévy super-diffusive systems, as well as to sub-diffusive systems [Sec. VI].

The effect of short-range cutoffs on the power-law interactions can easily be treated [Sec. VII]. We numerically explore our results on domain profiles [Sec. VIII, see also Fig. 6]. Finally, we conclude [Sec. IX].

Throughout this paper we concentrate on exponents, or scaling dependencies, of various quantities in the latetime limit. Inequalities apply to exponents, so that a process is dominant if it is asymptotically largest as $t \rightarrow$ $\infty$. We denote the mechanisms with a subscript $D, F$, or $B$ for diffusive, local force driven, or long-range ballistic processes, respectively.

\section{LONG-RANGE INTERACTIONS}

We work in the overdamped limit, in which particle velocity equals the applied force times a constant mobility, $\eta$. The pairwise forces are

$$
f_{i j}=(n-1) C q_{i} q_{j} / r_{i j}^{n}
$$

between particles with charges $\left\{q_{i}\right\}$ and pairwise separations $\left\{r_{i j}\right\}$. This corresponds to a pairwise interaction energy $E_{i j}=C q_{i} q_{j} r_{i j}^{1-n}$, with a logarithm at $n=1$. Thus the particle velocity is

$$
\partial_{t} \vec{r}_{i}=\eta \sum_{j \neq i} f_{i j} \hat{r}_{i j}+\vec{\phi}_{i}
$$

where we have added a random uncorrelated noise for diffusive motion with diffusion constant $D$, where $\left\langle\vec{\phi}_{i}(t)\right.$. $\left.\vec{\phi}_{j}\left(t^{\prime}\right)\right\rangle=D \delta_{i j} \delta\left(t-t^{\prime}\right)$, When oppositely charged particles get within a fixed capture radius $r_{c}$, they annihilate instantaneously 21]. We are interested in the behavior of the system at late times when distinct length-scales are well-separated.

\section{A. Initial Conditions}

Most studies of reaction diffusion with long-range interactions have focused on the case of random uncorrelated initial conditions, where the particles are randomly placed with a local Poisson distribution 10 14, 16, 18, 17, 18. Experimentally, it is more natural to quench a system of charged particles from a hightemperature state in which they are in thermal equilibrium [1],15]. 
Charge fluctuations may be usefully characterized by the typical excess charge density at scale $L$,

$$
\delta \rho \sim L^{-\mu} .
$$

This can be thought of as the excess charge density in a region of size $L$ after coarse-graining to that scale. It is also related to the magnitude of the Fourier-component $\rho_{1 / L}$ of the charge-density by

$$
\left|\rho_{1 / L}\right| \sim L^{d / 2-\mu},
$$

as obtained by summing up $O\left(L_{\infty}^{d} / L^{d}\right)$ uncorrelated contributions of size $L^{d} \delta \rho(L)$ and normalizing out the constant contribution due to the system size, $L_{\infty}$. As a result we can obtain $\mu$ directly from $\rho_{k}$.

We are only interested in the scale dependence of initial charge fluctuations that survive for long times - i.e. for fluctuations at large scales and correspondingly small $k$. Hence we consider a continuum charge-density $\rho(r)$ with energy

$$
\begin{aligned}
E & =C / 2 \int d^{d} r d^{d} r^{\prime} \rho(r) \rho\left(r^{\prime}\right) /\left|r-r^{\prime}\right|^{n-1} \\
& =C / 2 \int \frac{d^{d} k}{(2 \pi)^{d}} \rho_{k} \rho_{-k} \epsilon_{k} .
\end{aligned}
$$

From $\epsilon_{k} \equiv \int d^{d} x e^{i k \cdot x} x^{1-n}$, we obtain $\epsilon_{k}=$ $\pi^{d / 2}(k / 2)^{n-1-d} \Gamma[(d+1-n) / 2] / \Gamma[(n-1) / 2]$ for $1<$ $n<d+1$. For $n \geq d+1$ we must introduce a UV cutoff, the inverse particle separation [22], which determines a leading $\epsilon_{k}=$ const small- $k$ behavior. For $n=1$ the interaction in Eqn. (5) should be logarithmic, and we get $\epsilon_{k}=\pi^{d / 2} 2^{d-1} k^{-d} \Gamma(d / 2)$. Imposing equipartition on Eqn. (5) with temperature $T$, we get $\left\langle\rho_{k} \rho_{-k}\right\rangle \sim k_{B} T / \epsilon_{k}$.

This derivation reproduces the small $k$ behavior of a more complete variational approach, see, e.g. [23]. Entropy factors do not affect the leading long-wavelength fluctuations, so that the integrand in Eqn. (5) is correct for small $k$. This means that $\rho_{k}$ is Gaussian distributed for $k \rightarrow 0$, and $\left\langle\left|\rho_{k}\right|\right\rangle \sim \epsilon_{k}^{-1 / 2}$. Comparing with Eqn. (四) we obtain

$$
\mu=\left\{\begin{array}{lr}
(2 d+1-n) / 2 & 1 \leq n<d+1 \\
d / 2 & n>d+1 .
\end{array}\right.
$$

This is illustrated in Fig. 11. Note that $d / 2 \leq \mu \leq$ $d$, so that long-range interactions always suppress initial charge-fluctuations. The maximal suppression is achieved at $n=1$ when $\mu=d$. [For $n<1$ we expect higher-point correlations to be significant.] For sharp enough interactions, with $n>d+1$, we recover uncorrelated initial conditions with $\mu=d / 2$.

The charge excess at scale $L$, as discussed above, is quite different from the charge excess within a sharp boundary of scale $L$, for example inside a sphere of radius $L$. The later has been proposed as a measure of charge fluctuations, particularly in Coulombic systems in general dimensions, with $n=d-1$, where a Gauss's law applies (see, e.g.. [1, 15, 16]) and in systems with topological defects 2426 where similar integral identities apply to the topological charge density. At high temperatures the charge inside a given closed surface is proportional to the square-root of the surface area, as obtained from integrating the appropriate random high-temperature field over the surface [26]. Taken literally this would imply that $\mu=(d+1) / 2$ [15], i.e. larger charge fluctuations than indicated in Eqn. (6) for Coulombic interactions. However a sharp surface picks up charge fluctuations at short scales in addition to the desired large-scale fluctuations. Indeed, the Coulombic or high-temperature nature of the system is moot - arbitrary sharp surfaces within a system with microscopic charge heterogeneities (e.g. atoms or thermal fluctuations) pick up charge fluctuations proportional to the square-root of the surface area. The useful and appropriate charge fluctuations at scale $L$ are given by Eqn. (6), which are not mixed with charge-fluctuations at short-scales [27].

\section{B. Domain Profile and Currents}

Charge fluctuations, coarse-grained to scales much larger than typical particle separations, decay via charged currents. These currents can be diffusive, or can be driven by the long-range interactions. We first consider segregated systems, where the domain size, $L(t)$, sets the scale of surviving charge fluctuations. The average density within a domain, $\bar{\rho} \sim l_{A A}^{-d}$ is simply proportional to the initial fluctuations at the domain scale, $\delta \rho(L)$, so that 15

$$
L \sim l_{A A}^{d / \mu} .
$$

Charge fluctuations at scales much larger than $L$ cannot have relaxed since charge transport at larger scales is cutoff by the domain structure. We now assume [28] that the domain profile is only determined by the average density, $\bar{\rho}$, and size, $L$, of domains. For example, domains have a typical density profile

$$
\begin{aligned}
\rho(r) & =l_{A A}^{-d} f(r / L), \\
& \sim l_{A A}^{-d}(r / L)^{\alpha},
\end{aligned}
$$

where $r$ is measured from the edge of the domain. The second equation holds near a domain edge, with $r \ll L$. We also require $r \gg W$ so that the profile is probed well away from the reaction zone. This scaling form has been proposed by Leyvraz and Redner in diffusive systems [7], and was numerically confirmed in $d=1$.

The assumption of an invariant scaled domain morphology is powerful, and is sufficient to determine the coarse-grained current density $J$ near the domain edge. The flux must have a dominant non-zero constant contribution near the domain edge for $r \ll L$ arising from the evolution of domain density, since no annihilation takes place in the domain interior. Consider the net charge 
of a domain, $Q \sim L^{d} \bar{\rho} \sim L^{d-\mu}$. It implies a non-zero net flux density $J \sim \dot{Q} / L^{d-1} \sim L^{1-\mu} / t$ near the domain edge. The exponent characterizing the domain profile, $\alpha$, is constrained to allow the dominant $J$ to be finite but non-zero for $r / L \rightarrow 0^{+}$.

If the dominant current is diffusive, then $J_{D} \sim \bar{\rho} / L$, so that

$$
J_{D} \sim L^{-(1+\mu)} .
$$

Imposing a constant diffusive current condition at the domain edge implies a linear profile, with $\alpha=1$. This agrees with studies of domain profiles in diffusive systems [7].

If the dominant current is driven by long-range forces then it is given by the coarse-grained field times the local charge density, $J_{F} \sim \rho(r) F(r)$. The field, $F(r)$, at a distance $r$ away from the domain edge due to the charge distribution given by Eqn. (8) is

$$
F(r) \sim \int_{l_{A A}(r)}^{L} d^{d} x[\rho(r+x)-\rho(r-x)] / x^{n} .
$$

We have used the domain scale as the long-distance cutoff, and the local interparticle spacing, $l_{A A}(r) \sim$ $\rho(r)^{-1 / d}$, as the short-distance cutoff. We also restrict the angular integral to be close to the normal direction from the interface, which retains the scaling behavior of $F(r)$ without requiring detailed information about the domain shape.

For $d>n$, charges at distances of order $L$ determines $F$, so that $F \sim \bar{\rho} L^{d-n} \sim L^{d-n-\mu}$. For a constant flux $J_{F}$ at small $r$, we must have $\rho(r) \sim$ const. (i.e. $\alpha=0$ ) so that $J_{F} \sim \bar{\rho}^{2} L^{d-n} \sim L^{d-n-2 \mu}$. When $d>2 n$, with uncorrelated initial conditions $(\mu=d / 2), F$ increases with the upper-cutoff of the integral in Eqn. (10), so that we should use the system-size, $L_{\infty}$, rather than $L$. For this case, the system size enters the dynamics, and the thermodynamic limit does not exist [10,11.

For $d<n$, the local charge distribution dominates the $F(r)$ integral. We can expand the density for $x \ll r$ and find $F(r) \sim \rho(r)^{(n-1) / d} r^{-1}$ for $n>d+1$. For $d<n<$ $d+1$, on the other hand, the integral is dominated by scales around $r$, and we find $F(r) \sim r^{\alpha+d-n} /\left[l_{A A}^{d} L^{\alpha}\right]$. Insisting on $J_{F}$ approaches a non-zero constant near the domain edge, we obtain Table If. These results apply far from the reaction zone, but otherwise close to the domain edge: $W \ll r \ll L$. [We determine the reaction zone width in Sec. IIE.] We see that currents dominantly driven by power-law interactions lead to non-trivial domain profiles, with $0 \leq \alpha \leq 1 / 2$, in dramatic contrast to the diffusive case where $\alpha=1$. It is also interesting that the current, $J_{F}$, has the long-range form for $d<n<d+1$, even though $\alpha \neq 0$.

When both long-range forces and diffusive effects are present, then we must compare $J_{F}$ and $J_{D}$, and identify which is larger at late times. We summarize the results in Fig. 目 for random uncorrelated initial conditions. For equilibrated high-temperature initial conditions $J_{D}$ is always asymptotically larger at late times.

\section{Particle Annihilation}

In a well mixed region of the system, where the typical spacing between oppositely charged particles is $l_{A B}$, what is the scaling of the particle lifetime $\tau\left(l_{A B}\right)$ ? There are three annihilation mechanisms: diffusive annihilation $\left(\tau_{D}\right)$, local interaction-driven annihilation $\left(\tau_{F}\right)$, and ballistic annihilation $\left(\tau_{B}\right)$. We determine their scaling dependence on $l_{A B}$ and hence identify the dominant mechanism at late times. Our essentially microscopic approach also provides insight into the applicability of coarse-grained treatments (see Sec. IIF).

In the force-free case, particles move diffusively with diffusion constant $D$, and annihilate with oppositely charged particles when they approach within a fixed distance $r_{c}$. In $d \leq 2$, trajectories are space filling and $\tau_{D} \sim l_{A B}^{2} / D$ - the time it takes for a particle to diffuse $l_{A B}$. For $d>2, \tau_{D} \sim l_{A B}^{d} /\left(D r_{c}^{d-2}\right)$, since each particle must explore the characteristic volume per particle to find an anti-particle to annihilate. We have

$$
\tau_{D} \sim \begin{cases}l_{A B}^{2} & d \leq 2, \\ l_{A B}^{d} & d>2 .\end{cases}
$$

In the diffusion-free case, considering only local interactions, $f \sim r^{-n}$, between two particles initially separated by $l_{A B}$, the annihilation time

$$
\tau_{F} \sim l_{A B}^{n+1} .
$$

For many particles in a region, the same result follows from the scaling of the velocities in Eqn. (2).

With both diffusion and local interactions, diffusion $\left(\tau_{D}\right)$ dominates the annihilation time for $n>1$, while for $n<1$ the force $\left(\tau_{F}\right)$ does. This follows directly from the particle dynamics, Eqn. (2). Rescale all distances by $l_{A B}$, and rescale time by $l_{A B}^{2}$, so that diffusion is unchanged in the scaled coordinates as $l_{A B}$ increases. Scaled velocities due to the force are then multiplied by $l_{A B}^{1-n}$. As a result, forces do not asymptotically contribute for $n>1$, while forces dominate for $n<1$. For $d>2$, this leads to the initially counter-intuitive result that diffusion dominates for $1<n<d-1$, even though $\tau_{D} \gg \tau_{F}$. This is a well-known result for $n=2$ [29]. Essentially, the competition between diffusion and the attractive interaction is along the separation vector between two particles, and hence is always one-dimensional in character. Indeed, in $d=1$ the faster annihilation mechanism dominates, and the marginal value is $n=1$. We have confirmed these predictions for various $n$ in $d \leq 3$ by placing a particle and an anti-particle in a periodic box, and plotting the average annihilation time as a function of the box size. In Fig. 3, we show our results for $d=3$. For $n>1$, the long-range force merely changes the effective capture size, leaving $\tau_{D} \sim l_{A B}^{d}$. 
When an applied or non-local force $F$ is present, we must also consider ballistic annihilation. With a velocity proportional to $F$, and in a time $\tau_{B}$, particles sweep out a volume proportional to $\tau_{B} F l_{*}^{d-1}$, where $l_{*}$ is the radius of the effective capture cross-section. Equating this to the typical volume $l_{A B}^{d}$ per particle, we obtain the typical ballistic annihilation time

$$
\tau_{B} \sim l_{A B}^{d} l_{*}^{1-d} / F .
$$

When no noise is present, a particle will be captured by local interactions only at separations less than $l_{*}$ where local interactions are as large as $F$, so that $F \sim f \sim 1 / l_{*}^{n}$ or $l_{*} \sim F^{-1 / n}$. We can use the typical forces $F(r)$ from Table If but must evaluate them at the reaction-zone width $W$ (see below, Sec. IIE) - since the reactionzone itself is neutral at a coarse-grained scale. Because $F$ depends on the system morphology, this requires a selfconsistent solution [30]. The results are simple: for $n>d$ we find $l_{*} \gtrsim l_{A B}$ and the dominant time-scale is $\tau_{F}$, while for $n<d$ we find $l_{*} \lesssim l_{A B}$ and so the dominant time-scale is $\tau_{B}$. This corresponds to the naive phase-space result from Eqn. (10) that charges from far away dominate local forces, and hence $\tau_{B}$ dominates, only when $n<d$.

When diffusive noise is present, we first ignore the local interactions and only consider an applied field $F$. For $d>2$, both the random walk and the ballistic force sweep out volume proportional to time. Because $F$ decreases with time, $\tau_{D}$ always asymptotically dominates. However, for $d \leq 2$ random walks recur, and the ballistic drift can enhance the volume covered by the random walk by suppressing the recurrence. The rate of volume swept out by the drifting particle, is a sphere of radius $l_{*}$ every $\Delta t \sim l_{*} / F \sim l_{*}^{2} / D$. This implies $l_{*} \sim 1 / F$, and leads to $\tau_{B} \sim l_{A B}^{d} F^{d-2}$.

When both noise and local interactions are present, the local capture cross-section $l_{*}$ is determined by the dominant non-ballistic process - i.e. $\tau_{D}$ for $n>1$ and $\tau_{F}$ for $n<1$. The shortest annihilation time for uncorrelated initial conditions is given in Fig. 1 . For equilibrated initial conditions, $\tau_{D}$ always dominates for $n>1$.

Our treatment of particle annihilation is essentially microscopic rather than coarse-grained, since we have built the particle separation, $l_{A B}$, directly into the annihilation times. We have derived our results by considering particle pairs, though we have included some multi-particle effects by never allowing particles to "escape" further than $l_{A B}$ from an anti-particle. We apply the results in mixed regions of the system such as reaction zones.

\section{Domain Morphology: Segregated or Mixed}

We assume the system is described by one of two morphologies [31], depending on whether coarse-grained charge fluctuations are comparable to or much less than the mean particle density at late times. The former case describes a segregated morphology with domains of particles separate from domains of antiparticles. In the second case, there is a mixed morphology with no clearly defined domains. We can use the dominant flux $J$ and fastest annihilation time $\tau$ to see which morphology is consistent. The system turns out to have a unique consistent morphology: either mixed or segregated.

First consider a segregated morphology. The system has domains of scale $L$ separated by reaction zones of width $W$. Within the zones there is a typical particle spacing $l_{A B}$. We ignore correlations or structure within the zones [32]. Our self-consistency constraints are that $l_{A B} \lesssim l_{A A}$, and that $W \lesssim L$. We impose the former because annihilation takes place in the reaction zone but not in the domain bulk, so the density in the reaction zone should be smaller as a result. We impose the latter since if it were not the case, with $W \gg L$, the system would be effectively all reaction zone and of a mixed morphology. These constrain the maximum rate that reaction zones can "process" incoming particles: the average density of particles that are in reaction zones, $W L^{d-1} /\left(l_{A B}^{d} L^{d}\right)$, is at most $1 / l_{A A}^{d}$. Conversely, the maximum annihilation rate is $1 / \tau\left(l_{A A}\right)$, since $\tau(l)$ is monotonic. Combining these, the maximum annihilation rate from reaction zones is $\partial_{t} \bar{\rho}_{\text {max }} \sim-1 /\left[l_{A A}^{d} \tau\left(l_{A A}\right)\right]$. For segregated systems, the actual rate of particles annihilating per unit volume is determined by the currents entering the reaction zones: $\partial_{t} \bar{\rho}_{\text {seg }} \sim-J L^{d-1} / L^{d} \sim-J / L$.

If the maximum reaction rate $\partial_{t} \bar{\rho}_{\max }$ asymptotically dominates the actual rate $\partial_{t} \bar{\rho}_{\text {seg }}$, then a domain structure is consistent. Indeed, since $\partial_{t} \bar{\rho}_{\max }$ is the rate of density decrease in a mixed morphology, the inequality indicates that the background density decay quickly with respect to the charge fluctuations - i.e. that the segregated structure occurs when it is consistent. If the maximum reaction rate is less than the particle flux, then segregated domains cannot be sustained, and the mixed morphology should result.

For the segregated morphology, we use $\partial_{t} \bar{\rho} \sim-J / L \sim$ $-\bar{\rho} / t$ and $L \sim l_{A A}^{d / \mu}$ to extract the domain scale and particle density within the domain.

For the mixed morphology, the flux does not drive annihilation of the mean density. Rather every particle is effectively in a reaction zone and has a characteristic lifetime $\tau$. Comparing this to the scaling of the density evolution, $\partial_{t} \bar{\rho} \sim-\bar{\rho} / t \sim-\bar{\rho} / \tau$, indicates that $\tau\left(l_{A A}\right) \sim t$.

The resulting growth law regimes are shown in Fig. 5 for systems with either uncorrelated or high-temperature equilibrated initial conditions, and with either force-only or force-and-noise dynamics. The exponents are summarized in Tab. I1. The noise-only case reproduces the Ovchinnikov-Zeldovich-Toussaint-Wilczek result [1], and is given in Fig. 同 b) by the short-range $(n \rightarrow \infty)$ limit of the force-and-noise dynamics with uncorrelated initial conditions.

We can now consider the consistency of the mixed morphology. The system has no domain structure, and has 
local charge separation scales $l_{A B} \sim l_{A A}$. Coarse-grained charge fluctuations, above a scale $X(t)$, remain from the initial conditions. The mixed morphology is consistent if the charge density of the remaining charge fluctuations $\delta \rho(X) \sim X^{-\mu}$ is much less than the mean particle density $\bar{\rho} \sim l_{A A}^{-d}$.

Given the scaling of net currents $J(X)$, the charge fluctuations evolve with $\partial_{t} \delta \rho(X) \sim \delta \rho / t \sim J X^{d-1} / X^{d} \sim$ $J / X$. For diffusive currents, $J_{D}(X) \sim \delta \rho(X) / X \sim$ $X^{-(\mu+1)}$, and we find that $X \sim t^{1 / 2}$. For force driven currents, $F(X) \sim X^{d-n-\mu}$ from Eqn. (10) using $X$ as the upper and lower cutoff. The force drives the net charge density $\delta \rho(X)$, and yields a net current $J_{F}(X) \sim$ $X^{d-n-2 \mu}$, leading to $X \sim t^{1 /(\mu+n+1-d)}$. Using the results for $l_{A A}$ from above, we confirm that $\delta \rho(X) \ll \bar{\rho}$ within the mixed regions of Fig. 5 .

Our exponents apply at regime boundaries, where they are continuous. However our approach does not determine any logarithmic factors. [Indeed, logarithmic factors are expected at $n=d$, whenever $J_{F}$ or $\tau_{B}$ dominates, due to the logarithmic divergence of the integral for $F$, Eqn. (10).] At a boundary between mixed and segregated regimes, any logarithms present determine the dominant morphology through the self-consistent approach described above. Without logarithms, the mixed morphology applies on the boundary, since $W \sim L$ there. The width $W$ characterizes the mean-distance to annihilation for particles, and thus there is a finite density of anti-particles any finite multiple of $W$ into a domain. When $W \sim L$, there is a finite density of anti-particles arbitrarily deep within a domain - i.e. the system is mixed. This is the case in the diffusion only case at $d=4$, which mixes [2].

\section{E. The Reaction Zone}

For reaction-diffusion systems with a segregated morphology, much progress has been made on the structure and evolution of the reaction zones between domains [3]. With long-range interactions, we develop a similar approach that balances the dominant flux into the reaction zone with the dominant annihilation mechanism within the reaction zone. This balance determines the reaction zone width, $W(t)$, and the interparticle spacing within the reaction zone, $l_{A B}(t)$. To simplify our discussion, we take the density of both species to be uniform throughout the reaction zone 32].

When a particle enters the reaction zone, it travels a typical distance $W$ before it annihilates - the "annihilation mean-free path". For a segregated system, $W$ must characterize the width of the reaction zone. A reactionzone that grows less rapidly than $W$ allows almost all particles to pass through unannihilated. On the other hand, a reaction-zone that grows more rapidly than $W$ would provide infinite number of annihilation mean freepaths as $t \rightarrow \infty$, and would not have a mix of particles and anti-particles at the far edge of the reaction-zone. Since slower or faster growth is not self-consistent, $W(t)$ characterizes the typical reaction zone width.

In the annihilation time $\tau$, a particle diffuses a typical distance $W_{D} \sim(D \tau)^{1 / 2}$, or it ballistically moves $W_{B} \sim F \tau$ under an applied field $F$, where $F$ is given by Table 1 . In any case, a particle must move at least the interparticle spacing to annihilate, $W_{F} \sim l_{A B}$. The largest of these widths describes how far a particle travels before annihilation, and so characterizes the width of the reaction-zone.

The reaction lifetime, $\tau$, the particle spacing in the reaction-zone, $l_{A B}$, and the zone width, $W$, are determined self-consistently. Given the dominant flux $J$, we equate the overall flux density into reaction zones $\dot{N}_{\text {flux }} \sim J L^{d-1} / L^{d}$ with the rate of annihilation within the reaction zones $W L^{d-1} /\left[L^{d} l_{A B}^{d} \tau\left(l_{A B}\right)\right]$. We choose the largest $W$ for the given $\tau$, and the fastest $\tau$ for the given $l_{A B}$. These dominate the width and annihilation, respectively. This provides a self-consistent solution for $\tau, l_{A B}$, and $W$.

For diffusion-only systems in $d \leq 2$, our argument is essentially that of Leyvraz and Redner [7]. We recover their results of $W \sim l_{A B} \sim t^{3 / 8}$, and $t^{1 / 3}$ in $d=1$ and 2 , respectively. For $d>2$, however, we have $W \sim \sqrt{\tau_{D}} \sim$ $l_{A B}^{d / 2}$ since random-walks are no longer space-filling, and we obtain $l_{A B} \sim t^{5 / 18}$ and $W \sim t^{5 / 12}$ in $d=3$, and $l_{A B} \sim l_{A A} \sim 1 / 4$ and $W \sim L \sim t^{1 / 2}$ in $d=4$. Our results coincide with the RG scaling results of Lee and Cardy [3]. We also recover their scaling relations with respect to currents, whenever $\tau_{D}$ dominates annihilation. In particular, with $\partial_{t} \bar{\rho} \sim J / L \sim W /\left(L l_{A B}^{d} \tau_{D}\right), \tau_{D}$ from Eqn. (11), and $W_{D} \sim \sqrt{\tau_{D}}$, we have $W_{D} \sim J^{-1 / 3}$ and $l_{A B} \sim J^{-2 /(3 d)}$ for $d>2$ and $W_{D} \sim l_{A B} \sim J^{-1 /(d+1)}$ for $d<2$. These relations hold independently of the process that dominates the currents.

Even within the mixed morphology, it is interesting to consider the distance $W$ a particle travels in a lifetime $\tau$. This distance $W(t)$ is the same scale as the remaining charge fluctuations, $X(t)$, as discussed before in the previous section. This is reasonable, since charge fluctuations at scales much larger than $W$ cannot be flattened out by charge motion. We summarize our results in Tab. III.

\section{F. Discussion}

In all cases the density decays more slowly as $n$ increases and the potential becomes sharper and hence shorter ranged. If noise is present, then diffusive processes eventually dominate as $n$ increases. As the spatial dimension $d$ is increased for any $n$, then a mixed morphology is eventually reached. For generalized Coulombic systems, with $n=d-1$, the density decays as $\bar{\rho} \sim 1 / t$ in all combinations of high-temperature or uncorrelated initial conditions and force and/or diffusive processes. They 
are always of mixed morphology.

With equilibrated initial conditions, diffusive processes dominate independently of $n$ when they are present. This is reasonable, since the high-temperature initial conditions balances the interactions with temperature. Temperature then becomes more relevant as it is reduced during the quench.

For systems without diffusive processes, the growth regimes and processes for uncorrelated and hightemperature initial conditions, in Fig. f. a) and d) respectively, correspond. The various growth exponents differ only due to the different initial charge fluctuations, characterized by $\mu$. On the other hand, with both diffusive and long-range processes the regimes do not correspond, between Fig. 5 b) and e), because the competition between force and diffusive processes depends on the charge fluctuations through $\mu$.

Exponents are continuous at regime boundaries for $l_{A A}, l_{A B}, L, \tau$, and $W$. For segregated systems $L \gtrsim$ $W \gtrsim l_{A B} \gtrsim l_{A A}$, where the last inequality holds since reactions should decrease the particle density. We also check that $\tau \lesssim t$ in all cases. In $d=1$, we check $W \sim l_{A B}$, as expected since the reaction zone is precisely one $\mathrm{AB}$ pair. At the border between mixed and segregated morphologies, the reaction zone is maximal, i.e. $W \sim L$ and $l_{A B} \sim l_{A A}$.

The particle density at the edge of a domain, at $r \sim W$ in Eqn. (8), scales the same as the particle density within the reaction zone, $l_{A B}^{-d}$. i.e. that $l_{A B}^{-d} \sim l_{A A}^{-d}(W / L)^{\alpha}$. Interesting special cases occur where $J_{F}$ dominates the flux but $W_{D}$ dominates the reaction zone width (regions $\left[i i^{4}\right]$ and $\left.\left[i i^{5}\right]\right)$. In these special cases, the singular domain profile, with $\alpha<1$, leads to a diverging diffusion flux as the domain edge is approached, which must eventually dominate at some distance $L_{X}$ from the domain edge. In particular, $L_{X}$ is determined by $\rho\left(L_{X}\right) / L_{X} \approx J_{F}$. Using the domain profile, Eqn. (8), we have $L^{-\mu-\alpha} L_{X}^{\alpha-1} \sim J_{F}$. If $L_{X} \ll W$, then the cross-over is pre-empted by the reaction zone and we do not expect to observe it [33]. However, precisely when $J_{F}$ and $W_{D}$ dominate we find that $W_{D} \ll L_{X} \ll L$, so that a linear transition regime is expected for $W_{D} \ll r \ll L_{X}$. The intermediate linear regime, when it occurs, does not change the density evolution or reaction-zone structure, since $J_{F}$ still characterizes the flux.

In reaction-diffusion systems, $d_{c}$ is the critical dimension above which a coarse-grained reaction rate $\lambda \rho_{A} \rho_{B}$ applies, and $d_{u}$ can be defined as the dimension above which we can neglect inhomogeneities. Below $d_{c}$ the reaction term is not given by $\rho^{2}$ in a coarse-grained description, and local density fluctuations must be taken into account [3, 8 . Above $d_{u}$ we can ignore spatial gradients and have $\partial_{t} \bar{\rho} \sim-\bar{\rho}^{2}$ and hence $\bar{\rho} \sim 1 / t$ (see [1]). Both of these effects stem from the diffusive annihilation mechanism. The local annihilation rate $\rho / \tau_{D}$ is proportional to $\rho^{2}$ only for $d>2$ where $\tau_{D} \sim \rho^{-1}$. This sets $d_{c}=2$. The mixed state is found for $d \geq 4$, so that $d_{u}=4$.

When only long-range interactions are included, these definitions are not as useful since neither $\tau_{F}$ nor $\tau_{B}$ are in general proportional to $\rho^{-1}$. As an example, when only force-driven evolution is included, as in Fig. 5 a) and d), the density in the mixed state has a $d$-dependent exponent, see $[i i i]$ and [vii]. Even the long-wavelength charge fluctuations remain relevant to drive the dominant ballistic annihilation. We could describe this as $d_{c}=$ $d_{u}=\infty$.

When diffusive and long-range processes are included, however, we see from Tab. III that the diffusive process $\tau_{D} \sim l_{A B}^{d}$ dominates above $d_{c}=2$, so that the critical dimension is unchanged from the diffusion-only case. In mixed morphologies diffusive annihilation always dominates, so that $d_{u}$ equals the dimension where the mixed morphology starts. For uncorrelated initial conditions we find $d_{u}=n+1$ for $n<3$, while $d_{u}=4$ above. For hightemperature initial conditions $d_{u}=(n+3) / 2$ for $n<5$, while $d_{u}=4$ above. Since diffusive processes dominate all regimes in Fig. 0 e), it is only through the suppression of initial charge fluctuations that the long-range interactions modify $d_{u}$.

We have assumed that the domains of the segregated morphology are characterized only by a size $L$ and characteristic particle separation $l_{A A}$, and that the reaction zones are similarly characterized by a width $W$ and a particle separation $l_{A B}$. This leads to the exponents summarized in the tables and figures. It remains possible that other lengths enter into the asymptotic evolution, for instance through fluctuations in the shape of domain boundaries (see, e.g., [8]). Our results are most robust for $n<d$, where only the domain scale $L$ enters in the calculation of the forces and currents. Because of that, we obtain the same result for the evolving density in a segregated morphology after a coarse graining to $O(L)$, and any new but shorter lengths would not change this result. It remains possible, however, that additional lengths change the structure of the reaction zone, and hence change the boundary between segregated and mixed morphologies. Similarly, higher point correlation effects may lead to more intermediate morphologies within what we label the mixed regime. For $n>d$ additional lengths would affect the evolution of the density, since short scales enter into the calculation of the characteristic forces and currents.

Spatial fluctuations of coarse-grained quantities have also been neglected in our treatment. We assume that fluctuation effects are not strong enough to change our results for exponents. Another way to view fluctuations is in terms of the distribution of various quantities. We effectively assume that distributions have negligible tails.

We have also not included the motion of domain boundaries, either from local heterogeneities in domain density, or from differing mobilities of the particle species. Domain motion should not affect the scaling exponents if the boundaries move slowly enough for the domain profile $\alpha$ to maintain itself. A simple check is reas- 
suring. The flux required to move domain interfaces, $J_{\text {motion }} \sim \bar{\rho} \dot{L} \sim \bar{\rho} L / t$, has the same scaling behavior as the characteristic flux, $J \sim L \partial_{t} \bar{\rho}$. Hence domain wall motion should introduce no new scales.

\section{PREVIOUS LONG-RANGE WORK}

Some of the earliest work on charged $A+B \rightarrow$ systems with long-range interactions was by Toyoki 10 . He treated uncorrelated initial conditions and force-only evolution - corresponding to Fig. 5 a). He presented the mean field analysis of the mixed morphology, our region $[i i i]$. By considering the mean-square force on a particle, he recovered the system-size dependence for $n<d / 2$. He also initiated numerical studies of $d=2$ systems. However he used a short-scale cutoff proportional to the average particle separation $l_{A A}$ (see Sec. VII), which hampers interpretation of his results. Jang et al. [16] numerically studied $d=2$ systems with $n=1$ and obtained $\bar{\rho} \sim t^{-0.55}$ using a large noise amplitude, and $\bar{\rho} \sim t^{-0.90}$ using a large force amplitude. This is consistent with our results of $\bar{\rho} \sim t^{-1 / 2}$ and $\bar{\rho} \sim t^{-1}$ for the pure noise or pure force systems, respectively.

Ispolatov and Krapivsky [1] focused on force-only evolution in $d=1$, with a segregated morphology. They obtained results consistent with ours for $n<d$, where their assumption of a constant domain profile, with $\alpha=0$, is valid (see Table II and Sec. VIII).

Ginzburg, Radzihovsky, and Clark [12 treat coarsegrained hydrodynamic systems with uncorrelated initial conditions and with both diffusion and long-range forces, for $n \geq d-1$. Our particle-based treatment can be seen as complementary to their work. We obtain the same results for density evolution, but we also obtain details about the reaction zone - including $W, l_{A B}$, and $\tau$. We also identify the mixed or segregated morphology of the system. Furthermore, the system-size dependence in the force for $n<d / 2$ 10,11 is missed in their treatment. It would be interesting to extend their approach to include the particle nature of the charges, possibly with new phenomenological annihilation terms. Burlatsky et al. 13] present a simple scaling model that obtains the same results as the self-consistent approach of Ginzburg et al. 12.

\section{ELECTRONIC SYSTEMS}

Asymptotic non-geminate pair-recombination in clean crystalline semiconductor systems should be described by our approach with $n=2$ and equilibrated hightemperature initial conditions. In $d=3$, we find $\bar{\rho} \sim t^{-1}$ with a mixed morphology. This result applies for every combination of uncorrelated or high-temperature initial conditions and force and/or diffusion. This is because all annihilation mechanisms have the same scaling, $\tau \sim l_{A B}^{3}$.
In contrast, for $d<3$ the annihilation mechanisms differ and the morphologies are all segregated. The structure of the evolving charge-fluctuations is thus much richer. Specifically, in $d=1$, and 2 we expect regime [viii $\left.{ }^{\prime}\right]$ to apply asymptotically with $\bar{\rho} \sim t^{-1 / 4}$ and $t^{-3 / 4}$ respectively. The decay rate is dramatically slowed due to the segregated morphology.

Clean $d=2$ systems exist in quantum hall effect (QHE) devices. QHE devices with skyrmion charge excitations (see, e.g., 34) may be particularly good systems to study these effects, due to their low mobilities and slow dynamics combined with sensitive time-resolved probes of their particle density. We will develop this in more detail in a separate publication 35, paying particular attention to the scaling of the amplitudes and the resulting pre-asymptotic cross-overs in the evolution.

\section{PARTICLE-LIKE TOPOLOGICAL DEFECTS}

Point-like topological defects, e.g. hedgehogs in $d=3$ or vortices in $d=2$, are found in liquid crystals and in vector $O(N)$ systems in $d=N$ dimensions. Considered pairwise, these defects have power-law interactions. Indeed, early theoretical work [10 on these systems was based on the interactions of point-like topological defects. However the evolution of systems with point-like topological defects is different than the dynamics of Sec. II (see however [36]). The order parameter field that supports topological defects provides a scale-dependent mobility to particle motion. Additionally, the mobility depends on the local environment, i.e. the interaction between defects is not a two-point particle-particle interaction. We may, however, take that as a first approximation. For $d=2 \mathrm{XY}$ or nematic systems, the mobility scales logarithmically with the particle velocity, $\eta(l) \sim 1 / \ln (d l / d t)$ [19]. For $d=3 O(3)$ or nematic systems, the mobility of hedgehogs scales as the inverse separation, $\eta(l) \sim 1 / l$ [37. In general, for $O(N)$ models in $N \geq 3$ dimensions, $\eta(l) \sim l^{2-N}$ 19. High-temperature equilibrated initial conditions are appropriate for quenches in physical systems. For point defects in $d \geq 3$, the effective interaction is linear [10] $(n=0)$, which has not been treated in this paper. However, $d=2$ systems are within our purview.

For the $d=2 \mathrm{XY}$ model, interactions between defects are logarithmic $(n=1)$. Ignoring the logarithmic mobility, and including equilibrated initial conditions [27], we have a mixed morphology with all lengths scaling the same, $l_{A A} \sim l_{A B} \sim L \sim W \sim t^{1 / 2}$. This is found whether or not diffusive processes are included, and indeed $\tau_{D} \sim \tau_{B} \sim \tau_{F} \sim l_{A B}^{2}$ and $J_{D} \sim J_{F} \sim L^{-3}$ for this system. The single length scale and the similarities between no noise quenches and quenches with diffusion matches the phase ordering results of the $d=2 \mathrm{XY}$ model 19,38 .

Two phase-ordering systems that do exhibit strong scaling violations are the $d=1$ XY model [39] and the 
$d=2 O(3)$ model 40 . Both of these systems support non-singular topological textures that have particle-like aspects. However topological textures have an intrinsic length-scale that evolves in time. Significant extensions to our approach would be necessary for these systems. We may also consider interacting topological defects in the patterned structures of driven (see, e.g., 41]) systems or of systems with competing interactions (see, e.g., 42). To apply our approach, the long-wavelength fluctuations $(\mu)$, the interaction $(n)$, the mobility $\eta(l)$, and the nature of any noise-driven transport must be identified.

\section{LÉVY SUPER-DIFFUSION}

Systems with long-range Lévy super-diffusion 43 have been used to model stirred reaction-diffusion systems. While there are no long-range interactions per se in these systems, super-diffusion enhances the reaction rate in a manner qualitatively similar to long-range interactions. Indeed, our methods can be applied to this case and agree with the results of Zumofen et al. 17] for the late-time evolution from uncorrelated initial conditions. We also obtain additional information about the reaction zone structure and interface profiles for segregated morphologies. For simplicity, we only consider uncorrelated initial conditions, with $\mu=d / 2$.

In the discrete formulation of Lévy flight, every particle hops a random distance $r$ along a random lattice direction with probability distribution $P(r) \propto r^{-1-\gamma}-$ annihilating any anti-particles it encounters along the way. We impose $1<\gamma<2$ so that the hop distribution has a finite first moment and is normalizable. The equivalent continuum dynamics is $\partial_{t} \rho_{k}=-D|k|^{\gamma} \rho_{k}$, where $\gamma>1$ is required.

In time $t$, taken to be large, a particle randomly Lévy walks a distance $R \sim t^{1 / \gamma}$. If $\gamma>d$, the volume bounding this walk $R^{d} \ll t$ and the walk is recurrent, or spacefilling. Conversely, if $\gamma<d$ the walk is sparse, and the particle explores a volume proportional to $t$ using its finite capture radius $r_{c}$. Paralleling Eqn. (11), the annihilation time in a well-mixed region of the system scales as

$$
\tau_{L} \sim \begin{cases}l_{A B}^{d} & \gamma<d \\ l_{A B}^{\gamma} & \gamma>d\end{cases}
$$

where the typical particle-antiparticle separation in the region is $l_{A B}$. From the Lévy flight, the net distance that the particles move before annihilating is $W \sim \tau_{L}^{1 / \gamma}$. For space-filling walks $(\gamma>d) W \sim l_{A B}$, while for sparse walks $(\gamma<d) W \sim l_{A B}^{d / \gamma} \gg l_{A B}$.

For a segregated morphology, there is a typical fluxdensity leaving domains. The flux-density is most easily obtained in the discrete formulation, where it is simply the number of particles that pass through a site in each time-step. From the Lévy flight distribution, a fraction $p \sim 1 / x^{\gamma}$ of particles contributes to the current from a given distance $x$ away. Only particles along lattice directions contribute in a single time-step and, since $\gamma>1$, the flux is dominated by particles nearby compared to the domain scale. Hence, our current is $J(r) \sim \int_{0}^{r} d y[\rho(r+y)-\rho(r-y)] / y^{\gamma}$ a distance $r$ from a domain edge, where $W \ll r \ll L$. Using the domain profile from Eqn. (\$), and requiring that $J(r)$ approaches a constant near the domain edge we obtain a non-linear profile exponent, $\alpha=\gamma-1$, and $J_{L} \sim \bar{\rho} L^{1-\gamma}$ for the particle current.

For segregated systems, we equate the flux out of domains $\partial_{t} \bar{\rho} \sim J L^{d-1} / L^{d} \sim l_{A A}^{-(d+2 \gamma)}$ to the time derivative of the charge density $l_{A A}^{-d} / t$ to obtain $l_{A A} \sim t^{1 /(2 \gamma)}$ and $\bar{\rho} \sim t^{-d /(2 \gamma)}$. The domain size $L \sim t^{1 / \gamma}$. We can also obtain the rate of particle annihilation from the reaction rate within the reaction zones, $\partial_{t} \bar{\rho} \sim W L^{d-1} /\left[L^{d} l_{A B}^{d} \tau_{L}\right]$, and compare to obtain

$$
l_{A B} \sim \begin{cases}t^{(d+2 \gamma-2) /[2 d(2 \gamma-1)]} & \gamma<d, \\ t^{(d+2 \gamma-2) /[2 \gamma(\gamma+d-1)]} & \gamma>d .\end{cases}
$$

Since particles are randomly Lévy-walking before annihilation, the reaction zone width is $W \sim l_{A B}$ for $d<\gamma$, and $W \sim l_{A B}^{d / \gamma}$ for $d>\gamma$.

The system has a segregated morphology, with $W \lesssim$ $L \sim l_{A A}^{2}$, for $\gamma>d / 2$. For $\gamma<d / 2$ the system is mixed, and the time-scale is set by the annihilation rate $\tau_{L} \sim t$, so that $l_{A A} \sim t^{1 / d}$ and $\bar{\rho} \sim 1 / t$. At $\gamma=d / 2$, the marginal case between segregated and mixed morphologies, we have $W \sim L$ and $l_{A B} \sim l_{A A}$. Our results agree with those of Zumofen et al. 17. We also obtain $\alpha, l_{A B}$, and $W$ characterizing the domain profile and reaction zone in segregated systems.

It is interesting that while our results are qualitatively similar to those obtained with long-range interactions, there is no effective interaction $n$ that recovers the growth-exponents for a given Lévy exponent $\gamma$. This is in contrast to the nonequilibrium steady-state properties of the kinetic Ising model, where Lévy flights generate effective long-range interactions 44 .

We may be able to apply these results to the motion of an single charged particle in a quenched random potential. This motion is sub-diffusive, with $\gamma>2$ and $R \sim t^{1 / \gamma}$, for potentials with sufficiently long-range correlations 45. One loop RG calculations by Deem and Park [18 for potentials caused by quenched Coulombic ions in $d=2(n=1)$, matched to the homogeneous evolution without any long-range interactions, indicate that sub-diffusive behavior alone may be sufficient to describe the evolution of the quenched system. If so, then our Lévy-flight results may be directly applied with the appropriate $\gamma$. However significant questions remain, such as the appropriate microscopic annihilation mechanisms for oppositely charged particles in a quenched random potential. 


\section{LONG-RANGE CUTOFFS}

It is interesting to explore quenched long-range systems numerically, but the computational burden can be large. Long-range cutoffs $L_{c u t}(t)$, usually on the order of the interparticle spacing, accelerate a simulation, but often at the cost of changing the physics.

Long-range cutoffs contribute in two places. The first is through the force integral driving particles fluxes, $F(r)$ in Eqn. (10). Any $L_{c u t} \ll L(t)$ replaces the upper-cutoff of the integral and change $F(r)$ for the cases where the integral is not dominated by short scales, i.e. for $n<d+$ 1. If $F(r)$ is changed, the domain profile exponents $\alpha$ are also affected. The second place cutoffs enter is through the annihilation time within the reaction zone, Eqn. (12). If the cutoff is smaller than the reaction-zone spacing, $L_{\text {cut }} \ll l_{A B}(t)$, then the annihilation dynamics of $\tau_{F}$ is changed. Ballistic annihilation times $\tau_{B}$ is qualitatively changed whenever $L_{c u t} \ll L(t)$. Amplitudes can also be affected by cutoffs, even when growth exponents are not.

As an illustration, we consider limiting interactions to nearest-neighbors. Consider uncorrelated initial conditions with no diffusion, Fig. 5 a). For nearest-neighbor interactions, we use $L_{c u t} \sim l_{A A}(r) \sim \rho(r)^{-1 / d}$, the local particle spacing. Putting $L_{c u t}$ instead of $L$ in Eqn. (10) leads to the same results as $n>d+1$ in Table 1 for all $n$. In particular, the reaction-zone profile is characterized by $\alpha=d /(n+d-1)$. The nearest-neighbor cutoff is of order $l_{A B}$ within the reaction zone so that local force-driven annihilation dynamics, with $\tau_{F}$, is qualitatively unchanged. The system size cannot enter the force integral, so there is no restriction on the interaction to $n>d / 2$. For a segregated morphology regime $[i]$ applies, while in the mixed regime $[i i i]$ does, though with $W \sim l_{A B}$ and annihilation through $\tau_{F}$. Comparing the particle annihilation rate $\partial_{t} \bar{\rho} \sim J / L$ to the maximum rate supported by reaction zones $\partial_{t} \bar{\rho}_{\max } \sim \bar{\rho} / \tau_{F}\left(l_{A A}\right) \sim 1 / l_{A A}^{n+d+1}$, we find that segregation occurs for all $d<3$. In summary, nearest neighbor interactions only leave the evolution qualitatively unchanged for $n>d+1$. A numerical test of the effects of nearest-neighbor interactions in $d=1$ is made in the next section.

\section{NUMERICAL INVESTIGATION}

We present some numerical results on the nontrivial domain profile exponent $\alpha$ in uncorrelated onedimensional systems without diffusion. We also consider the effect of a cutoff $L_{c u t}$ limiting interactions to nearestneighbors. More results will be presented in future work 35.

In $d=1$, we expect $\alpha=(n-1) / 2$ for $1<n<2$ (regime $\left[i i^{0}\right]$ ) and $\alpha=1 / n$ for $n>2$ (regime $[i]$ ). We studied systems with 8000 particles with $n=3 / 2[375$ samples] and $n=2$ [300 samples]. We expect $\alpha=1 / 4$ and $1 / 2$ respectively. We avoided finite-size effects by comparisons with size 4000 systems. We also considered systems with only nearest-neighbor interactions, which are expected to modify the domain profile for $n<2$, see Sec. VII In particular, we expect regime $[i]$ to apply for all $n<2$. For $n=3 / 2$, we expect $\alpha=2 / 3$. We studied systems with 128000 particles with $n=3 / 2[200$ samples] and $n=2$ [53 samples], and checked finite-size effects with systems of 32000 particles. We found results consistent with expectations. We show our results for the domain profiles in Fig. 6.

\section{CONCLUSIONS}

By considering the annihilation dynamics in well mixed regions of a charged particle system, and balancing the annihilation against currents driven by charge inhomogeneities left over from the initial conditions, we selfconsistently determine the morphology and evolution of quenched charged-particle systems with long-range interactions. We also contribute a visceral description of the dynamics. We characterize the system with the scale of domains $L$ and reaction-zones $W$, and the particle spacings within domains $l_{A A}$ and reaction-zones $l_{A B}$. For mixed systems $L \sim l_{A A} \sim l_{A B}$. Our results are summarized in Fig. 5, and in the three tables. Our primary assumption is that the lengths we have used are sufficient to characterize the evolving system. The scaling form for the domain profile, Eqn. (8), follows from this assumption.

The results of this paper will hopefully inspire more formal derivations and numerical tests, as well as experimental tests in electronic systems. Comparisons with existing treatments is encouraging, particularly agreement with the field-theory approach of Lee and Cardy [3] for reaction-diffusion systems, with the hydrodynamic treatment of Ginzburg, Radzihovsky, and Clark [12] for uncorrelated initial conditions with diffusion and longrange interactions, and with the Lévy super-diffusion results of Zumofen et al. [17]. There are also many new results pertaining to the reaction-zones, domain profiles, and systems with equilibrated high-temperature initial conditions.

Electronic systems $(n=2)$ in two-dimensions should provide an experimental test for our results. Asymptotically, we predict a segregated morphology with domain size $L \sim t^{1 / 2}$ and average density $\bar{\rho} \sim t^{-3 / 4}$ for photoexcited quantum-well or QHE systems (high-temperature equilibrium initial conditions, regime $\left[\right.$ viii $\left.\left.^{\prime} / v_{i i i^{\prime \prime}}\right]\right)$. This will be explored at more length in a separate publication [35]. If initial conditions with uncorrelated initial conditions can be manufactured, then we expect regime $\left[i v^{\prime} / i v^{\prime \prime} / i i^{4} / i i^{5}\right]$ to apply: a segregated morphology with $L \sim t^{1 / 2}$ and $\bar{\rho} \sim t^{-1 / 2}$. These contrast dramatically with the mixed morphology and density decay $\bar{\rho} \sim t^{-1}$ in three-dimensional electronic systems.

I thank the EPSRC for support under Grant No. 
GR/J78044, the NSERC, and le Fonds pour la Formation de Chercheurs et l'Aide à la Recherche du Québec. I also thank John Chalker, Slava Ispolatov, Ben Lee, Klaus Oerding, Zoltan Rácz, and Beate Schmittmann for useful discussions.

[1] A. A. Ovchinnikov and Ya. B. Zeldovich, Chem. Phys. 28, 215 (1978); D. Toussaint and F. Wilczek, J. Chem. Phys. 78, 2642 (1983).

[2] M. Bramson and J. L. Lebowitz, J. Stat. Phys. 62, 297 (1991); 65, 941 (1991).

[3] B. P. Lee and J. Cardy, Phys. Rev. E 50, R3287 (1994); J. Stat. Phys. 80, 971 (1995); 87, 951 (1997). Note that $\ell_{r z} \sim l_{A B}$.

[4] L. Gálfi and Z. Rácz, Phys. Rev. A 38, 3151 (1988)

[5] S. Cornell and M. Droz, Phys. Rev. Lett. 70, 3824 (1993).

[6] E. Ben-Naim and S. Redner, J. Phys. A 25, L575 (1992). Note that their coarse-grained approach is only valid for $d \geq 2$.

[7] F. Leyvraz and S. Redner, Phys. Rev. Lett. 66, 2168 (1991); Phys. Rev. A 46, 3132 (1992); S. Redner and F. Leyvraz, J. Stat. Phys. 65, 1043 (1991). They impose $W \sim l_{A B}$. For reaction-diffusive systems this is correct only for $d \leq 2$.

[8] M. Howard and J. Cardy, J. Phys. A 28, 3599 (1995).

[9] No diagrams dress the particle propagators of formal approaches when the only interaction is annihilation (see [3]). This is not true when long-range interactions are included.

[10] H. Toyoki in Dynamics of Ordering Processes in Condensed Matter, ed. by S. Komura and H. Furukawa (Plenum, New York, 1988) p. 173; Phys. Rev. A 42, 911 (1990).

[11] I. Ispolatov and P. L. Krapivsky, Phys. Rev. E 53, 3154 (1996). Note that their $\lambda \equiv n$.

[12] V. V. Ginzburg, L. Radzihovsky, and N. A. Clark, Phys. Rev. E 55, 395 (1997).

[13] S. F. Burlatsky, V. V. Ginzburg, and N. A. Clark, Phys. Rev. E 54, R1056 (1996).

[14] T. Ohtsuki, Phys. Lett. 106A, 224 (1984). This paper applies to the generalized Coulombic model $n=d-1$, rather than $n=2$ as stated.

[15] V. V. Ginzburg, P. D. Beale, and N. A. Clark, Phys. Rev. E 52, 2583 (1995).

[16] W. G. Jang, V. V. Ginzburg, C. D. Muzny, and N. A. Clark, Phys. Rev. E 51, 411 (1995).

[17] G. Zumofen, J. Klafter, and M. F. Shlesinger, Phys. Rev. Lett. 77, 2830 (1996).

[18] M. W. Deem and J.-M. Park, Phys. Rev. E 57, 2681 (1998); J.-M. Park and M. W. Deem, submitted to Phys. Rev. E.

[19] A. D. Rutenberg and A. J. Bray, Phys. Rev. E 51, 5499 (1995).

[20] When one species is immobile correlations can become important [f], particularly for the domain structure of the immobile species. For purely diffusive systems, this is a singular limit [3].

[21] There is no pair-creation, $\emptyset \rightarrow A+B$, in our dynamics. This corresponds to being at a temperature far below the chemical potential of pairs.

[22] The initial particle density is tuned by a chemical potential. It does not affect the asymptotic exponents of the subsequent evolution.

[23] P. M. Chaikin and T. C. Lubensky, Principles of Condensed Matter Physics (Cambridge, 1995).

[24] M. B. Einhorn, D. L. Stein, and D. Toussaint, Phys. Rev. D 21, 3295 (1980); C. Liu and M. Muthukumar, J. Chem. Phys. 106, 7822 (1997).

[25] M. Mondello and N. Goldenfeld, Phys. Rev. A 42, 5865 (1990).

[26] D. Dhar, Phys. Lett A 81, 19 (1981).

[27] A quenched 2d XY model can be approximated by a system of charged-vortices interacting logarithmically, with $n=1$ (see Sec. V). Coarse-grained charge fluctuations, as described by Eqn. (4), lead to the correct mixed morphology with $L \sim l_{A A} \sim t^{1 / 2}$ (up to logarithms, see, e.g., $1938)$. In contrast, characterizing the initial fluctuations by the excess number of vortices within a sharply defined box of size $L$, i.e. $\mu=3 / 2$, leads to a segregated morphology with $L \sim t^{1 / 2}$ but $l_{A A} \sim t^{3 / 8}$ [25, which is not observed 19,38.

[28] We coarse-grain the charge density to a small but finite fraction of $L$, and we assume a smooth domain structure at that scale. Alternatively, we could build in a fractal domain structure (see [i]), but we cannot a priori determine if a fractal structure evolves from the dynamics.

[29] L. Onsager, Phys. Rev. 54, 556 (1938).

[30] We self-consistently determine the shortest applicable annihilation time $\tau\left(l_{A B}\right)$, the largest relevant reaction zone width $W(\tau)$, and the corresponding particle spacing in the reaction-zone $l_{A B}$, in terms of their time-exponents. The results are given in Tables III and [II].

[31] Other morphologies are possible in principle, though they have not been observed in reaction-diffusion systems. They would introduce new length-scales.

[32] In general, reaction zones are inhomogeneous, with profiles scaled by the reaction zone width $W$ and particlespacing $l_{A B}$ [4, 6], and with dilute multi-scaling tails [8]. Our analysis is unchanged by these refinements.

[33] This linear transition zone is precisely the "boundary layer" in the mean-field treatment of Ben-Naim and Redner [6]. Note that force-driven and ballistic annihilation mechanisms, not contained in their approach, stabilize the reaction zone when $L_{X} \ll W$.

[34] S. E. Barrett et al., Phys. Rev. Lett 74, 5112 (1995).

[35] A. D. Rutenberg, unpublished.

[36] The ordering kinetics of scalar systems in $d=1$ with long range interactions reduces to alternatingly charged domain walls (particles) with long-range interactions. When the domain walls are initially randomly distributed see A. D. Rutenberg and A. J. Bray, Phys. Rev. E 50, 1900 (1994).

[37] A. Pargellis, N. Turok, B. Yurke, Phys. Rev. Lett. 67, 1570 (1991); L. M. Pismen and B. Y. Rubinstein, Phys. Rev. Lett. 69, 96 (1992). 
[38] B. Yurke, A. N. Pargellis, T. Kovacs, and D. A. Huse, Phys. Rev. E 47, 1525 (1993).

[39] A. D. Rutenberg and A. J. Bray, Phys. Rev. Lett. 74, 3836 (1995).

[40] A. D. Rutenberg, W. J. Zakrzewski, and M. Zapotocky, Europhys. Lett. 39, 49 (1997).

[41] M. C. Cross and D. I. Meiron, Phys. Rev. Lett. 75, 2152 (1995).

[42] C. Sagui and R. C. Desai, Phys. Rev. E 52, 2807 (1995).

[43] M. F. Shlesinger, G. M. Zaslavsky, and J. Klafter, Nature 363, 31 (1993).

[44] B. Bergersen and Z. Rácz, Phys. Rev. Lett. 67, 3047 (1991); H.-J. Xu, B. Bergersen, and Z. Rácz, Phys. Rev. E 47, 1520 (1993).

[45] J.-P. Bouchaud and A. Georges, Phys. Rep. 195, 127 (1990) and references therein.

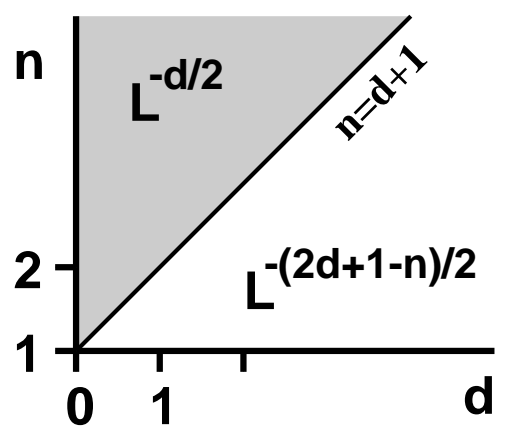

FIG. 1. Charge density fluctuations $\delta \rho \sim L^{-\mu}$ for equilibrated high-temperature initial conditions. In the shaded region the initial conditions are uncorrelated at large scales; in the unshaded region interactions reduce large-scale charge fluctuations.

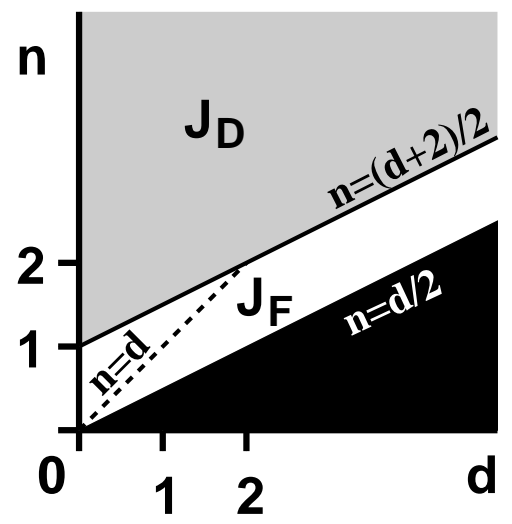

FIG. 2. The asymptotically dominant flux starting from uncorrelated initial conditions $(\mu=d / 2)$. When $J_{F}$ dominates, see Tab. If, when $J_{D}$ dominates see Eqn. (9). For equilibrated high-temperature initial conditions $J_{D}$ always dominates at late times.

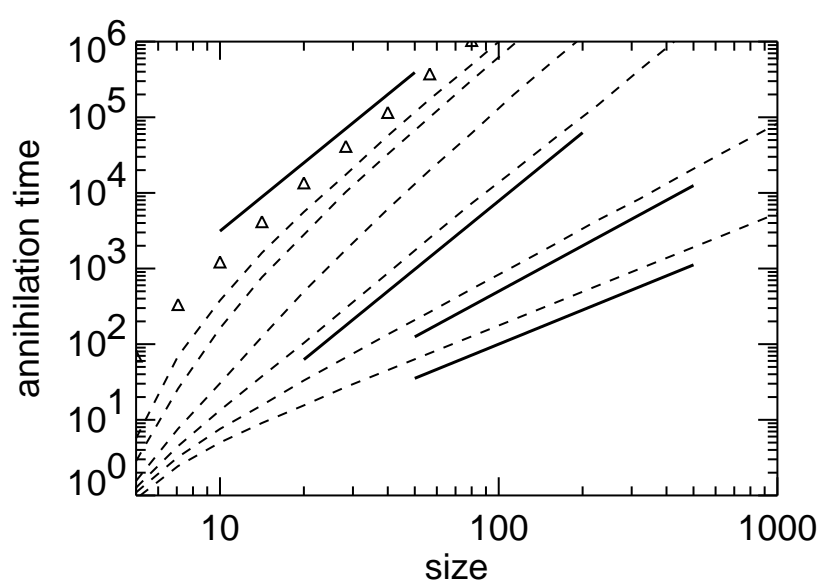

FIG. 3. Annihilation time $\tau(l)$ vs size $l$ of a particle/anti-particle pair placed in periodic boxes of size $l$ in $d=3$, where the particles are initially $l / 4$ apart. They evolve according to Eqn. (2) with a central force $f=1 / r^{n}$, mobility $\eta=1$, diffusion constant $D=1 / 4$, and time-step $\delta t=1$. The dashed curves correspond to, from bottom to top, $n=1 / 2,1$, $3 / 2,2,3$, and 4 . Triangles correspond to purely diffusive motion. The short solid lines indicate the expected asymptotic behavior, with $\tau(l) \sim l^{3 / 2}, l^{2}$, and $l^{3}$ (twice), from bottom to top respectively. Diffusion, with $\tau_{D} \sim l^{3}$, dominates asymptotically for $n \geq 1$.

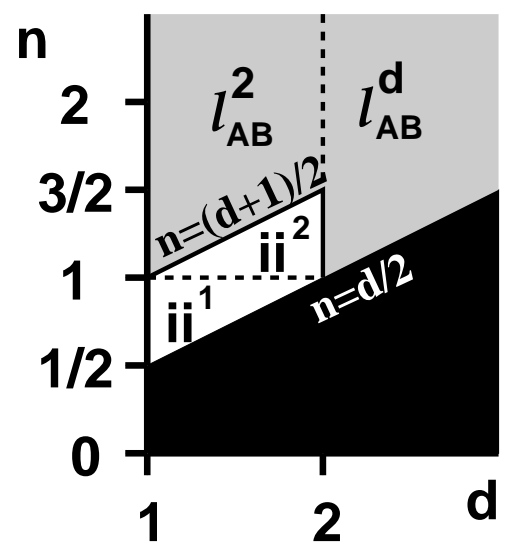

FIG. 4. Dominant annihilation times for force and noise, with uncorrelated initial conditions. Diffusive annihilation $\left(\tau_{D}\right)$ dominates in the shaded region, while ballistic annihilation $\left(\tau_{B} \sim l_{A B}^{d} F^{d-2}\right)$ dominates in the clear region. The regions $\left[i i^{1}\right]$ and $\left[i i^{2}\right]$ correspond to Fig. 5 . For equilibrium high-temperature initial conditions, when noise is present, $\tau_{D}$ always dominates. For force-only annihilation, irrespective of initial conditions, $\tau_{B} \sim l_{A B}^{d} F^{(d-n-1) / n}$ dominates for $n<d$ while $\tau_{F} \sim l_{A B}^{n+1}$ dominates for $n>d$. 
a)
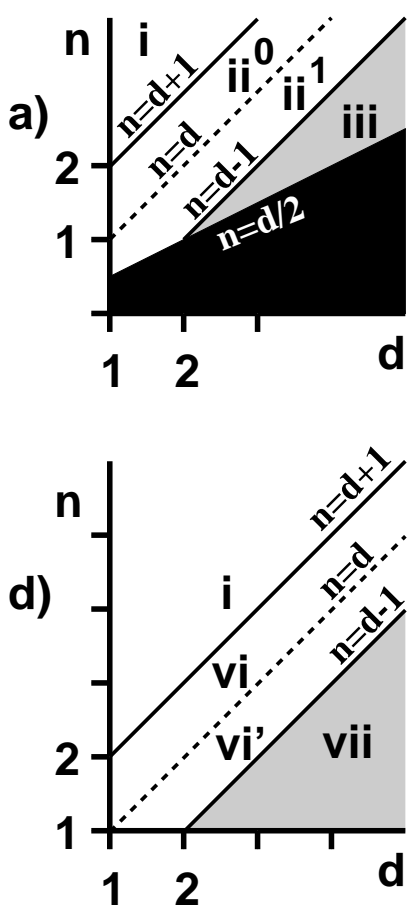

b)
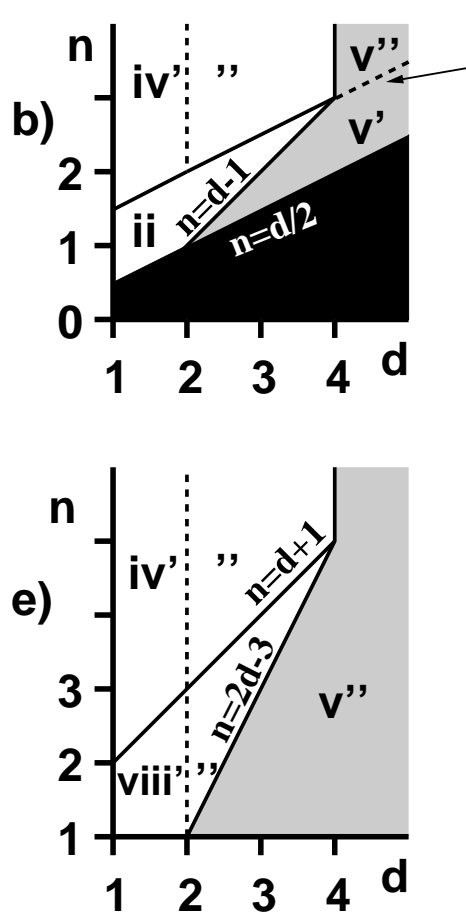

$n=(d+2) / 2$

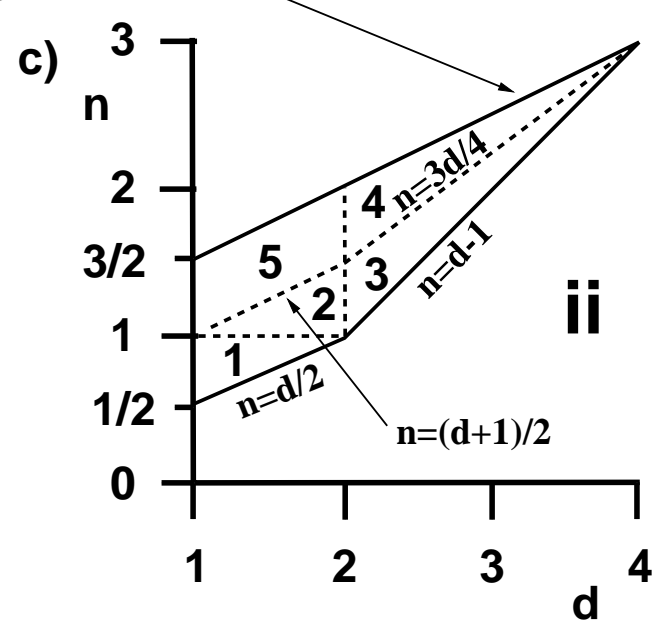

FIG. 5. Different regimes of density evolution. Blank regions indicate a segregated morphology, shaded a mixed morphology, while black indicates excluded regimes that have no thermodynamic limit. Dashed lines separate regimes, numbered with Roman numerals, with different reaction-zone structure (see Table III). The figures correspond to: a) uncorrelated initial conditions, force only, b) uncorrelated initial conditions, diffusion and force, c) enlargement of b), with the $\left[i i^{1}\right]$ to $\left[i i^{5}\right]$ subregions numbered, d) equilibrated high-temperature initial conditions, force only, and e) equilibrated initial conditions, noise and force.

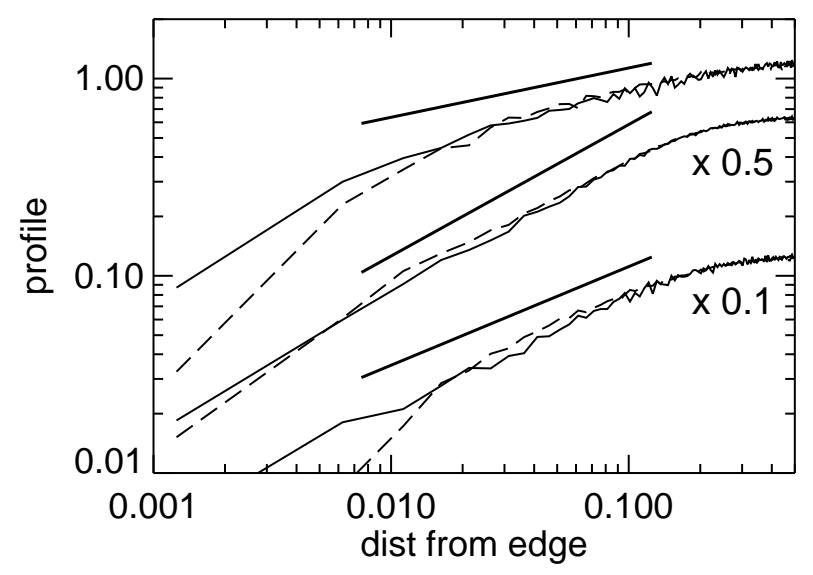

FIG. 6. Average domain profile $f(x)$ vs scaled distance $x$ from the edge of the domain. From top to bottom are $n=3 / 2$ (at $t=1.9 \times 10^{-5}$ dashed and 0.0013 solid $), n=3 / 2(t=0.0038$ and 0.09 for dashed and solid respectively) with nearest-neighbor interactions, and $n=2\left(t=1.9 \times 10^{-5}\right.$ and 0.25 respectively $)$ with nearest-neighbor interactions; all are in $d=1$. The second and third sets of curves have been scaled by 0.5 and 0.1 respectively. The straight segments indicate the expected domain profiles: $\alpha=1 / 4,2 / 3$, and $1 / 2$. The crossover at small $x$ is due to the reaction zone, and moves to smaller scaled distance at later times. 


\begin{tabular}{|l|ccc|}
\hline \hline & $\alpha$ & $F(r)$ & $J_{F}$ \\
\hline$n<d$ & 0 & $L^{d-n-\mu}$ & $L^{d-n-2 \mu}$ \\
$d<n<d+1$ & $(n-d) / 2$ & $r^{(d-n) / 2} L^{(d-n-2 \mu) / 2}$ & $L^{d-n-2 \mu}$ \\
$n>d+1$ & $d /(n+d-1)$ & $(L / r)^{d /(n+d-1)} L^{-(n+1) / 2}$ & $L^{-(n+d+1) / 2}$ \\
\hline \hline
\end{tabular}

TABLE I. Domain profile exponents, $\alpha$, coarse-grained field a distance $r \ll L$ from the domain edge, $F(r)$, and force-driven flux, $J_{F}$, for different interaction exponents $n$. When diffusive fluxes dominate $\alpha=1$.

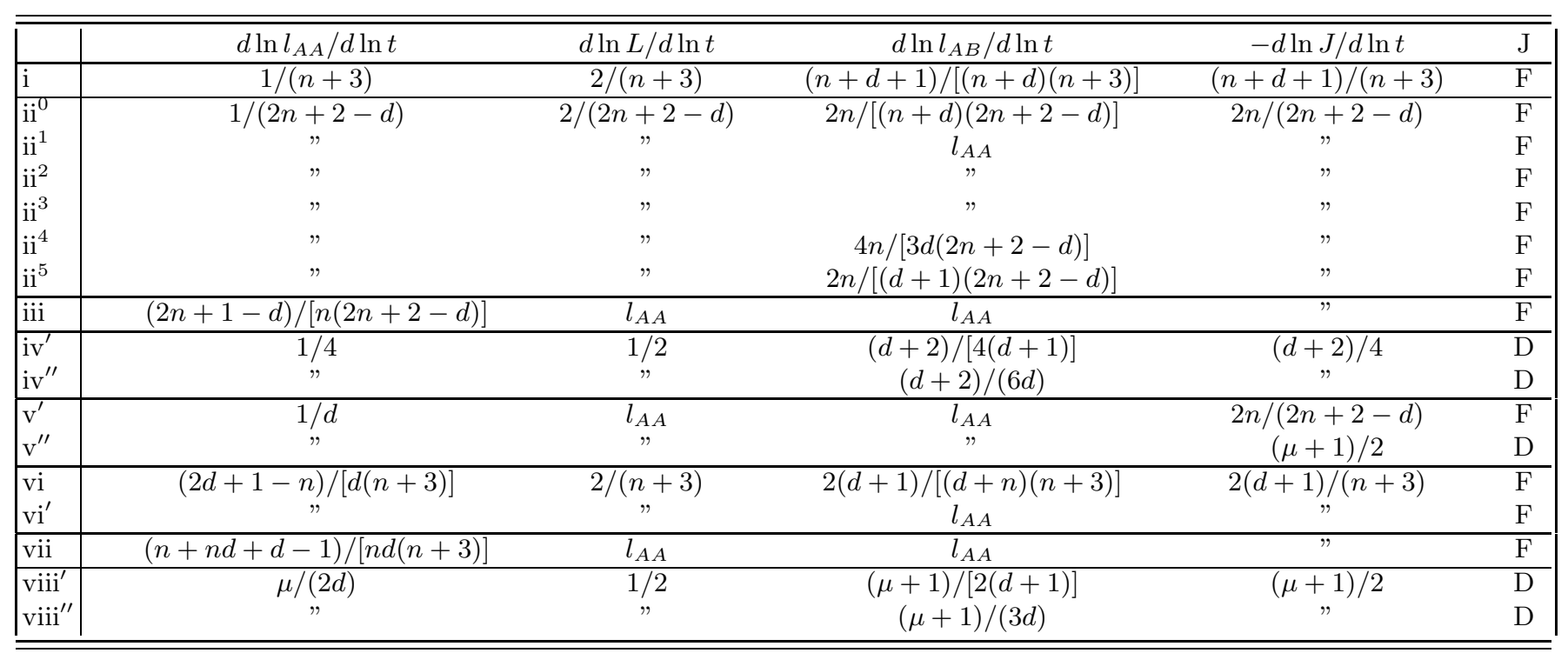

TABLE II. Growth laws of $l_{A A}, L, l_{A B}$, and $J$ for the different labeled regimes in Fig. 0 . Lengths with the same scaling as $l_{A A}$ are indicated. The last column indicates the dominant flux mechanism, where "D" is diffusive and " $F "$ is force driven.

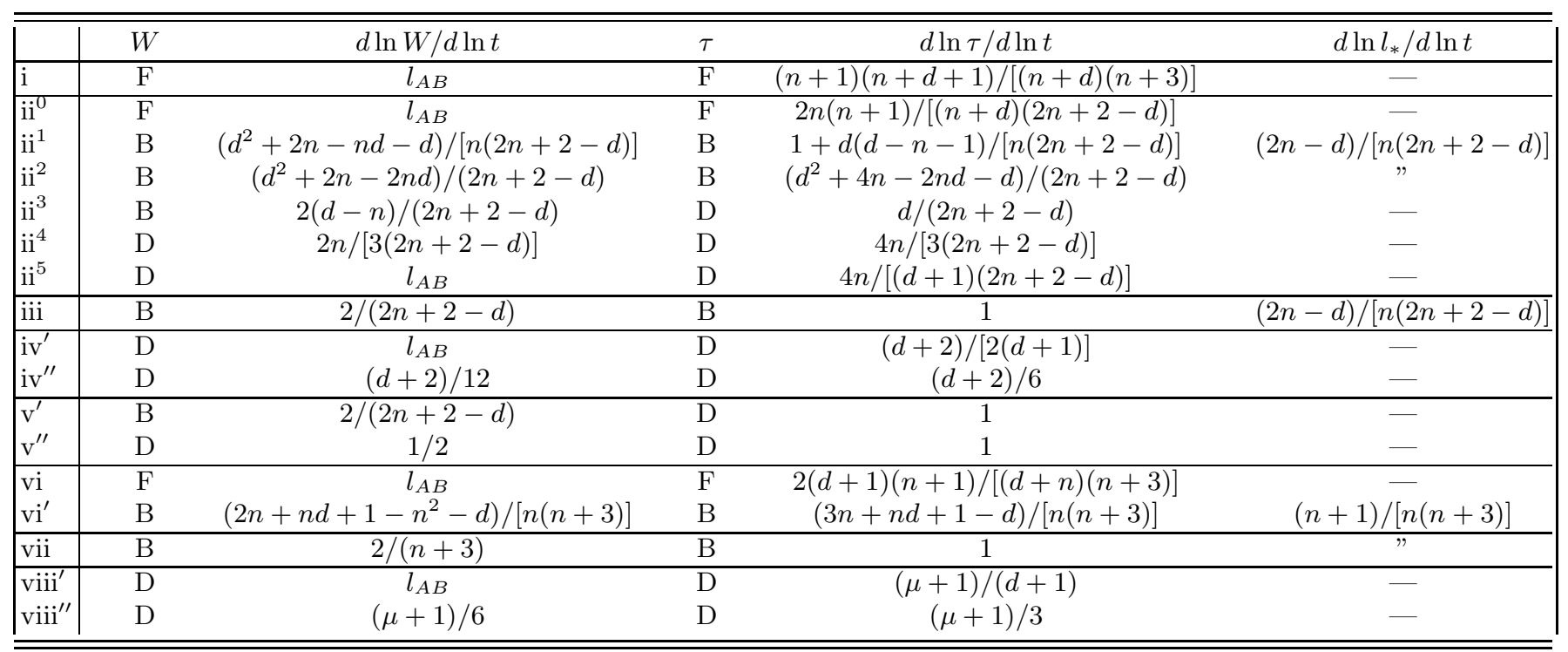

TABLE III. Growth laws of $W, \tau$, and $l_{*}$ for the different labeled regimes in Fig. 5. Reaction-zone widths $W$ with the same scaling as $l_{A B}$ are indicated. The dominant mechanisms determining $W$ and $\tau$ are also indicated, where "D" is diffusive, " $\mathrm{F}$ " is force driven, and "B" is ballistic. Note that when the morphology is mixed, $\tau \sim t$ and $W$ indicates the scale of remaining charge fluctuations. 